\title{
Minimal Representations of a Face of a Convex Polyhedron and Some Applications
}

\author{
Ta Van Tu ${ }^{1}$
}

Received: 4 December 2019 / Revised: 31 August 2020 / Accepted: 28 October 2020 /

Published online: 3 July 2021

(C) The Author(s) 2021

\begin{abstract}
In this paper, we propose a method for determining all minimal representations of a face of a polyhedron defined by a system of linear inequalities. Main difficulties for determining prime and minimal representations of a face are that the deletion of one redundant constraint can change the redundancy of other constraints and the number of descriptor index pairs for the face can be huge. To reduce computational efforts in finding all minimal representations of a face, we prove and use properties that deleting strongly redundant constraints does not change the redundancy of other constraints and all minimal representations of a face can be found only in the set of all prime representations of the face corresponding to the maximal descriptor index set for it. The proposed method is based on a top-down search strategy, is easy to implement, and has many computational advantages. Based on minimal representations of a face, a reduction of degeneracy degrees of the face and ideas to improve some known methods for finding all maximal efficient faces in multiple objective linear programming are presented. Numerical examples are given to illustrate the method.
\end{abstract}

Keywords Faces of a polyhedron · Degeneracy degrees of faces · Prime and minimal representations of a face - Maximal descriptor index set $\cdot$ Multiple objective programming

Mathematics Subject Classification (2010) 90C29 · 90C90 · 47N10 · 34M03

\section{Introduction}

Convex polyhedrons are widely used in theoretical and practical problems. Therefore, representations of a convex polyhedron play an important role in investigating and solving many problems. There are many ways for describing a convex polyhedron, but its main presentations are a H-representation (a representation by equalities or inequalities) and V-representation (a representation by finitely many points and directions). For details on V-representations and H-representations of a polyhedron, the readers can refer to Luan and

Ta Van Tu

t_vantu@yahoo.com

1 Department of Operations Research, Corvinus University of Budapest, 1093 Budapest, Hungary 
Yen [9], Ciripoi et al. [3], and Rockafellar [11]. In this paper, we investigate representations of faces of a convex polyhedron given by a H-representation

$$
a_{i} x \leq b_{i}, \quad i=1, \ldots, m,
$$

where $x \in \mathbb{R}^{n}, b_{i} \in \mathbb{R}, a_{i}^{T} \in \mathbb{R}^{n}$, and ${ }^{T}$ denotes vector or matrix transposition. For brevity of presentation, we shall use the following notation: For two vectors $y=\left(y_{1}, \ldots, y_{n}\right)^{T}$ and $z=\left(z_{1}, \ldots, z_{n}\right)^{T}, y \leq z$ if and only if $y_{i} \leq z_{i}$ for all $i=1, \ldots, n$; for two subsets $\Omega^{1}$ and $\Omega^{2}$ of a set, $\Omega^{1} \subset \Omega^{2}$ if and only if $\Omega^{1} \subseteq \Omega^{2}$ and $\Omega^{1} \neq \Omega^{2}$. Let $P$ be polyhedron $(1.1), \bar{I}=\{1, \ldots, m\} \backslash I$ and

$$
S(I, J)=\left\{\begin{array}{l|l}
x \in \mathbb{R}^{n} & \begin{array}{l}
a_{i} x=b_{i}, i \in I \\
a_{j} x \leq b_{j}, j \in J
\end{array}
\end{array}\right\} .
$$

A nonempty subset $F$ of $P$ is said to be a face of it if there is a subset $I \subseteq\{1, \ldots, m\}$ such that $F=S(I, \bar{I})$. Such a set $I$ is called a descriptor index set for $F$ and $S(I, \bar{I})$ is called a descriptor set for $F$ corresponding to $I$. An index pair $(I, J)$ such that $I, J \subseteq\{1, \ldots, m\}$ and $I \cap J=\varnothing$ is called descriptor index pair for a face $F$ if $S(I, J)=F$. An index set $I \in R E(F)$ is said to be a maximal descriptor index set for a face $F$, denoted by $I_{\max }$, if there is no $J \in R E(F)$ such that $I \subset J$, where

$$
R E(F)=\{J \subseteq\{1, \ldots, m\} \mid S(J, \bar{J})=F\} .
$$

A face $F$ is said to be degenerate if $|R E(F)| \geq 2$, where $|$.$| denotes the number of elements$ of a set. An index $i \in I$ or a constraint $a_{i} x=b_{i}$ is called redundant for $S(I, J)$ if $S(I)$ $\{i\}, J)=S(I, J)$. An index $j \in J$ or a constraint $a_{j} x \leq b_{j}$ is called redundant for $S(I, J)$ if $S(I, J \backslash\{j\})=S(I, J)$ and is called a strongly redundant for $S(I, J)$ if $a_{j} x<b_{j}$ for all $x \in S(I, J \backslash\{j\})$. We say that an index pair $(I, J)$ contains a redundant index for $S(I, J)$ if $I$ or $J$ contains at least one redundant index for $S(I, J)$. An index pair $\left(I^{1}, J^{1}\right)$ is called a weak reduction of $(I, J)$ if $S\left(I^{1}, J^{1}\right)=S(I, J), I^{1} \subseteq I$ and $J^{1} \subseteq J$. An index pair $(I, J)$ is called a prime representation of a face $F$ corresponding to a descriptor index set $K$ for $F$ if $(I, J)$ is a weak reduction of $(K, \bar{K})$ and contains no redundant indices for $S(I, J)$. A descriptor index pair $(I, J)$ for a face $F$ is called a prime representation of $F$ if $(I, J)$ contains no redundant indices for $S(I, J)$. A descriptor index pair $(I, J)$ for a face $F$ is called a minimal representation of the face $F$ if

$$
|I \cup J|=\min \{|K \cup M| \mid(K, M) \in T(F)\},
$$

where $T(F)$ is the set of all descriptor index pairs for the face $F$, defined as follows

$$
T(F)=\{(M, N) \mid S(M, N)=F ; M, N \subseteq\{1, \ldots, m\} \text { and } M \cap N=\emptyset\} .
$$

A minimal representation of a face is also a prime representation of this face and might be not unique.

The concepts of prime and minimal representations given here are generalizations of those previously investigated, for example, in Telgen [16], Boneh et al. [2], and Sierksma and Tijssen [15]. In order to find prime or minimal representations of a face, a removal of redundant indices from descriptor index pairs for the face must be done. A removal of some redundant indices from the index pair $(\emptyset,\{1, \ldots, m\})$ for the special face $S(\emptyset,\{1, \ldots, m\})$ is dealt with in Greenberg [7]. The difference between the cardinalities of any two prime representations of the special face $S(\emptyset,\{1, \ldots, m\})$ is investigated in Boneh et al. [2]. An optimized representation of a polyhedron investigated in Scholl et al. [14] is a representation of this polyhedron containing no redundant constraints. Thus, an optimized representation of a polyhedron in their concept only is a prime representation and, in general, is not a minimal representation of the polyhedron (see Property 6.3 and Remark 6.5 later). In addition, 
the method presented in [14] may fail to find all prime representations of a face. A minimal representation of a polytope considered in Klintberg et al. [8] is a representation of this polytope containing no redundant constraints. Therefore, it can happen that a minimal representation of a polytope considered in their work is not a minimal representation in the sense of [16]. Based on the methods of eliminating a redundant constraint given in Telgen [16], Scholl et al. [14], Fukuda [6], and Maréchal and Périn [10], finding one prime representation of a face is not hard; the difficulty lies in finding all prime and minimal representations of a face. The main difficulty for determining all prime and minimal representations of a face is that the deletion of one redundant constraint can change the redundancy of other constraints and the number of descriptor index pairs for the face can be large. This can cause huge computations in determining all prime and minimal representations of a face. For a given descriptor index set $J$ for a face, a necessary and sufficient condition for checking the index pair $(J, \bar{J})$ to be a minimal representation of this face can be found but no method for determining a minimal representation of a face is given in Telgen [16] when the index pair describing it is not a minimal representation of the face. Similarity transformations between minimal representations of a face are dealt with in Dam [4]. Some properties of minimal representations of a face can be found in, for example, Telgen [16], Boneh et al. [2], Dam [4], and Sierksma and Tijssen [15], but a method for determining a minimal representation of a face in a general case has not been found.

In this paper, we first propose a method for determining all minimal representations of a given face of a convex polyhedral set, then we show some applications of minimal representations of a face. Based on the definition of minimal representations of a face, all minimal representations of a face can be obtained by finding all prime representations of it. This method can cause huge computations in determining all minimal representations of a face. To reduce computational efforts for finding all minimal representations of a face, we prove and use properties that deleting strongly redundant constraints does not change the redundancy of other constraints and all minimal representations of a face can be obtained by finding only the set of all prime representations of the face corresponding to the maximal descriptor index set for it. In addition, a top-down search method is proposed for determining the set of all prime representations of a face corresponding to the maximal descriptor index set for it. This method is simple, is easy to implement, and has many computational advantages (see Remark 4.3 later). For applications of minimal representations of a face, we deal with a reduction of the number of constraints used to represent a face, a reduction of degeneracy degrees of a face and ideas to improve some known methods for finding all maximal efficient faces in multiple objective linear programming, and some known methods for optimizing a function over the efficient set.

This paper is organized as follows: Some properties of minimal representations of a face of a polyhedron are presented in Section 2. Determining all prime representations of a face corresponding to the maximal descriptor index set for it is dealt with in Section 3. An algorithm for determining all minimal representations of a given face and examples to illustrate the working of the algorithm are presented in Sections 4 and 5. Some applications of minimal representations of a face are considered in Section 6.

\section{Some Properties of Minimal Representations of a Face of a Polyhedron}

For brevity of presentation, through this paper, let $F$ be the face described by a descriptor index set $I$. A point $x^{0}$ of the face $F$ is called an inner point of it if there is $J \in R E(F)$ 
such that $a_{i} x^{0}<b_{i}$ for all $i \in \bar{J}$. From Tu [18, Property 2.6] it follows that every face has at least one inner point. An index $j \in K$ is called an implicit equality index for $S(J, K)$ if $a_{j} x=b_{j}$ for all $x \in S(J, K)$. From the definition of minimal representations of a face and Telgen [16, Theorem 4.1], the following property is easily obtained:

Property 2.1 An index pair $(J, K) \in T(F)$ is a minimal representation of the face $F$ if and only if $(J, K)$ contains no redundant indices and $K$ does not contain implicit equality indices for $S(J, K)$.

A relation between minimal representations and prime representations of a face is considered in the following property:

Property $2.2 R E_{\min }(F) \subseteq R E_{\text {prim }}\left(F, I_{\max }\right)$, where $R E_{\min }(F)$ and $R E_{\text {prim }}\left(F, I_{\max }\right)$ are the set of all minimal representations of the face $F$ and the set of all prime representations of the face $F$ corresponding to $I_{\max }$, respectively.

Proof We consider an arbitrary element $(J, K) \in R E_{\min }(F)$. From the definition of a minimal representation of a face and Property 2.1, it is easily seen that $J \cap K=\emptyset,(J, K)$ contains no redundant indices and $K$ contains no implicit equality indices for $S(J, K)$. If there is $j^{0} \in J \backslash I_{\max }$, then $j^{0} \in \bar{I}_{\max }$. From this, we have $a_{j^{0}} x^{0}<b_{j^{0}}$, where $x^{0}$ is an inner point of $F$. Thus, $x^{0} \notin S(J, K)$. This is a contradiction because $S(J, K)=F$. Therefore, $J \subseteq I_{\max }$. Assume that there is $k^{0} \in K \backslash \bar{I}_{\max }$. It is easily seen that $k^{0} \in I_{\max }$. Hence, $a_{k^{0}} x=b_{k^{0}}$ for all $x \in S\left(I_{\max }, \bar{I}_{\max }\right)$. Since $S\left(I_{\max }, \bar{I}_{\max }\right)=S(J, K), k^{0}$ is an implicit equality index for $S(J, K)$. This contradicts the fact that $K$ contains no implicit equality indices for $S(J, K)$. Thus, we also have $K \subseteq \bar{I}_{\max }$. Therefore, from the definition of a prime representation of a face corresponding to $I_{\max }$, it follows that $(J, K) \in R E_{\text {prim }}\left(F, I_{\max }\right)$. The proof is complete.

The set $R E_{\min }(F)$ can be obtained by determining all prime representations of $F$ but this method requires many computational efforts. Property 2.2 shows that only the set $R E_{\text {prim }}\left(F, I_{\max }\right)$ is needed. Now, we deal with another important property of the set $R E_{\text {prim }}\left(F, I_{\max }\right)$.

Property 2.3 For an arbitrary element $(J, K) \in R E_{\text {prim }}\left(F, I_{\max }\right)$, the index pair $(J, K)$ contains no redundant indices and the index set $K$ does not contain implicit equality indices for $S(J, K)$.

Proof From the definition of a prime representation of a face, it is clear that $(J, K)$ contains no redundant indices for $S(J, K)$. Assume that there is $j \in K$ such that $j$ is an implicit equality index for $S(J, K)$. Hence, we have $\min \left\{a_{j} x \mid x \in S(J, K)\right\}=b_{j}$. Since $S(J, K)=S\left(I_{\max }, \bar{I}_{\max }\right), \min \left\{a_{j} x \mid x \in S\left(I_{\max }, \bar{I}_{\max }\right)\right\}=b_{j}$. Noting that $K \subseteq \bar{I}_{\max }$, we have $j \in \bar{I}_{\max }$. Thus, $j$ is an implicit equality index for $S\left(I_{\max }, \bar{I}_{\max }\right)$. Hence, $F=S\left(I_{\max }, \bar{I}_{\max }\right)=S\left(I_{\max }, \bar{I}_{\max }\right) \cap\left\{x \in \mathbb{R}^{n} \mid a_{j} x=b_{j}\right\}=S\left(I_{\max } \cup\{j\}, \bar{I}_{\max } \backslash\{j\}\right)$. Thus, $\left\{I_{\max } \cup\{j\}\right\} \in R E(F)$. This contradicts the definition of the maximal descriptor index set for the face $F$. Therefore, $K$ does not contain any implicit equality indices for $S(J, K)$. The proof is complete.

Based on Property 2.3, we can obtain a result stronger than that in Property 2.2 . 
Theorem 2.4RE $R E_{\min }(F)=R E_{\text {prim }}\left(F, I_{\max }\right)$.

Proof From Properties 2.1 and 2.3, it follows that $R E_{\min }(F) \supseteq R E_{\text {prim }}\left(F, I_{\max }\right)$. Therefore, from Property 2.2, we have $R E_{\min }(F)=R E_{\text {prim }}\left(F, I_{\max }\right)$. The proof is complete.

It is clear that $R E_{\text {min }}(F) \subseteq R E_{\text {prim }}(F)$, where $R E_{\text {prim }}(F)$ is the set of all prime representations of the face $F$. Let $R E_{\text {prim }}(F, J)$ be the set of all prime representations of a face $F$ corresponding to $J \in R E(F)$. The following corollary shows a condition for equality in this inequality:

Corollary 2.5 If the face $F$ is not degenerate, then $R E_{\min }(F)=R E_{\text {prim }}(F)$.

Proof Since $F$ is not degenerate, $R E(F)=\left\{I_{\max }\right\}$. Therefore, from Theorem 2.4, it follows that $R E_{\text {prim }}(F)=\cup\left\{R E_{\text {prim }}(F, J) \mid J \in R E(F)\right\}=R E_{\text {prim }}\left(F, I_{\text {max }}\right)=R E_{\text {min }}(F)$.

Based on Theorem 2.4, we only need to find the set $R E_{\text {prim }}\left(F, I_{\max }\right)$ for determining all minimal representations of the face $F$.

\section{Determining All Prime Representations of a Face Corresponding to the Maximal Descriptor Index Set}

Let $i q(J, K)$ be the set of all implicit equality indices for $S(J, K)$. The set $I_{\max }$ can be determined on the basis of an index set $i q(I, \bar{I})$ found by solving $|\bar{I}|$ linear programming (LP) problems

$$
\min \left\{a_{i} x \mid x \in S(I, \bar{I})\right\},
$$

and $i q(I, \bar{I})=\left\{i \in \bar{I} \mid o^{i}=b_{i}\right\}$, where $i \in \bar{I}$ and $o^{i}$ is the optimal value of (3.1). Another method for determining the sets $i q(I, \bar{I})$ and $I_{\max }$ is shown in Tu [19] by solving only one LP problem:

Property 3.1 If $I \in R E(F)$ and $\left(x^{0}, z^{0}(\bar{I}), y^{0}, y^{0}(\bar{I}), \alpha^{0}\right)^{T}$ is an arbitrary feasible solution of $P M(I)$ with $\alpha^{0}>0$, then

(i) $\operatorname{iq}(I, \bar{I})=I Q\left(I, x^{0}\right)$,

(ii) $I_{\max }=I \cup i q(I, \bar{I})$,

where I $Q\left(I, x^{0}\right)=\left\{i \in \bar{I} \mid z_{i}^{0}(\bar{I})=0\right\}, P M(I)$ is the LP problem:

$$
\begin{aligned}
& \max \alpha \\
& A(I) x=b(I), \\
& A(\bar{I}) x+z(\bar{I})=b(\bar{I}), \\
& y^{T}(I) A(I)+y^{T}(\bar{I}) A(\bar{I})=0, \\
& y^{T}(I) b(I)+y^{T}(\bar{I}) b(\bar{I})=0, \\
& z(\bar{I})+y(\bar{I})-\alpha e(\bar{I}) \geq 0, \\
& z(\bar{I}) \geq 0, y(\bar{I}) \geq 0,
\end{aligned}
$$

$A(J)$ is the matrix obtained from the matrix A of the left hand side of (1.1) by deleting rows whose indices are not in $J ; b(J)$ is the vector obtained from the vector $b$ of the right hand 
side of (1.1) by deleting components whose indices are not in $J ; y(J), z(J)$ and $e(J)$ are similarly defined from vectors $y \in \mathbb{R}^{m}, z \in \mathbb{R}^{m}$ and $e=(1, \ldots, 1)^{T} \in \mathbb{R}^{m}$, respectively.

An efficient algorithm for determining the set $I_{\max }$ is presented in Subroutine $\operatorname{INDEXFACE}(I, \alpha, \beta)$ in [19]. Let

$$
\begin{aligned}
& r(J)=\operatorname{rank}\left\{a_{j} \mid j \in J\right\}, \\
& P(j, K, L) \text { be a problem } \max \left\{a_{j} x \mid x \in S(K, L)\right\}, \\
& a_{j}^{*}(K, L)= \begin{cases}b_{j}+1 & \text { if there is } x^{0} \in S(K, L) \text { such that } a_{j} x^{0}>b_{j}, \\
a_{j}^{\text {opt }}(K, L) & \text { if } P(j, K, L) \text { has an optimal solution, }\end{cases}
\end{aligned}
$$

where $a_{j}^{o p t}(K, L)$ is the optimal value of $P(j, K, L)$. It is clear that if $(K, L)$ is a weak reduction of the index pair $(J, \bar{J})$ corresponding to an arbitrary element $J \in R E(F)$, then $S(K, L) \neq \emptyset$ and $a_{j}^{*}(K, L)$ exists. In addition, the problem $P(j, K, L)$ need not be solved to optimality for determining $a_{j}^{*}(K, L)$ if there is $x^{0} \in S(K, L)$ such that $a_{j} x^{0}>b_{j}$.

Some simple conditions for the redundancy of an index pair are given in the following property whose proof is easily obtained from the definitions of redundant indices and the Gaussian elimination:

Property 3.2 (i) An index $k \in K$ is redundant for $S(K, L)$ if and only if $r(K)=r(K \backslash\{k\})$. (ii) An index $j \in L$ is redundant for $S(K, L)$ if and only if $a_{j}^{*}(K, L \backslash\{j\}) \leq b_{j}$.

From the definition of prime representations of a face and Property 3.2, we easily have the following property:

Property 3.3 If $J \in R E(F)$, then the index pair $(J, \bar{J})$ is a prime representation of the face $F$ if and only if $r(J)=|J|$ and $a_{j}^{*}(J, \bar{J} \backslash\{j\})>b_{j}$ for all $j \in \bar{J}$.

For an element $J \in R E(F)$, we define the following sets:

$$
\begin{aligned}
& \bar{J}^{1,1}=\left\{j \in \bar{J} \mid a_{j}^{*}(J, \bar{J} \backslash\{j\})<b_{j}\right\}, \\
& \bar{J}^{1,2}=\left\{j \in \bar{J} \mid a_{j}^{*}(J, \bar{J} \backslash\{j\})=b_{j}\right\}, \\
& \bar{J}^{1}=\bar{J}^{1,1} \bigcup \bar{J}^{1,2}, \\
& \bar{J}^{2}=\left\{j \in \bar{J} \mid a_{j}^{*}(J, \bar{J} \backslash\{j\})>b_{j}\right\}, \\
& T_{1}(F, J)=\{K \subseteq J|| K \mid=r(K) \text { and } r(K)=r(J)\}, \\
& T_{2}(F, J)=\left\{G \subseteq \bar{J}^{1} \mid a_{j}^{*}(J, \bar{J} \backslash G) \leq b_{j}, \forall j \in G\right\}, \\
& T_{3}(F, J)=\left\{G \in T_{2}(F, J) \mid \nexists G^{1} \in T_{2}(F, J): G \subset G^{1}\right\} .
\end{aligned}
$$

In order to determine the set $R E_{\min }(F)$, based on Theorem 2.4, it is enough to find the set $R E_{\text {prim }}\left(F, I_{\max }\right)$. Now, we consider a formula to compute the set $R E_{\text {prim }}\left(F, I_{\max }\right)$.

Theorem 3.4 $R E_{\text {prim }}\left(F, I_{\max }\right)=\left\{\left(K, \bar{I}_{\max } \backslash G\right) \mid K \in T_{1}\left(F, I_{\max }\right), G \in T_{3}\left(F, I_{\max }\right)\right\}$. 
Proof We will show that $\left(K, \bar{I}_{\max } \backslash G\right)$ is a prime representation of $F$ corresponding to $I_{\max }$ for every element $\left(K, \bar{I}_{\max } \backslash G\right) \in R E^{1}\left(F, I_{\max }\right)$, where

$$
R E^{1}\left(F, I_{\max }\right)=\left\{\left(K, \bar{I}_{\max } \backslash G\right) \mid K \in T_{1}\left(F, I_{\max }\right), G \in T_{3}\left(F, I_{\max }\right)\right\} .
$$

First, we will show that $S\left(K, \bar{I}_{\max } \backslash G\right)=F$. It is clear that $F=S\left(I_{\max }, \bar{I}_{\max }\right)=$ $S\left(I_{\max }, \bar{I}_{\max } \backslash G\right) \bigcap S(\emptyset, G)$. From the definition of the set $T_{2}\left(F, I_{\max }\right)$, it follows that $S\left(I_{\max }, \bar{I}_{\max } \backslash G\right) \subseteq S(\emptyset, G)$. Thus, $S\left(I_{\max }, \bar{I}_{\max } \backslash G\right)=F$. By a proof similar to that of [19, Property 5.2], it can be easily obtained that $S\left(K, \bar{I}_{\max } \backslash G\right)=F$. Consequently, from Property 3.2, it follows that $K$ does not contain any redundant indices for $S\left(K, \bar{I}_{\max } \backslash G\right)$. From the definition of $T_{3}\left(F, I_{\max }\right)$, it is clear that $\bar{I}_{\max } \backslash G$ does not contain any redundant indices for $S\left(K, \bar{I}_{\max } \backslash G\right)$. Thus, $\left(K, \bar{I}_{\max } \backslash G\right) \in R E_{\text {prim }}\left(F, I_{\max }\right)$ and $R E^{1}\left(F, I_{\max }\right) \subseteq R E_{\text {prim }}\left(F, I_{\max }\right)$.

Conversely, we consider an arbitrary prime representation $\left(I^{1}, I^{2}\right) \in R E_{\text {prim }}\left(F, I_{\max }\right)$ and will show that $\left(I^{1}, I^{2}\right) \in R E^{1}\left(F, I_{\max }\right)$. It can be easily seen that $I^{1} \subseteq I_{\max }, I^{2} \subseteq \bar{I}_{\max }$ and $\left(I^{1}, I^{2}\right)$ does not contain any redundant indices for $S\left(I^{1}, I^{2}\right)$. From Property 3.2, it follows that $r\left(I^{1}\right)=\left|I^{1}\right|$. Since $I^{1} \subseteq I_{\max }, r\left(I^{1}\right) \leq r\left(I_{\max }\right)$. We will show that $r\left(I^{1}\right)=$ $r\left(I_{\max }\right)$. Assume that $r\left(I^{1}\right)<r\left(I_{\max }\right)$. It is clear that there is $i^{0} \in I_{\max } \backslash I^{1}$ such that $r\left(I^{1} \cup\right.$ $\left.\left\{i^{0}\right\}\right)=\left|I^{1} \cup\left\{i^{0}\right\}\right|$. Since $r\left(I_{\max }\right) \leq n,\left|I^{1}\right|+1 \leq n$. Let $S M_{J^{1}}$ be a $\left(\left|I^{1}\right|+1\right) \times\left(\left|I^{1}\right|+1\right)$ square nonsingular submatrix of $\left(\begin{array}{l}A\left(I^{1}\right) \\ a_{i^{0}}\end{array}\right)$ and $d=\left(d_{1}, \ldots, d_{\left|I^{1}\right|+1}\right)^{T}$ be the $\left|I^{1}\right|+1-t h$ column of the inverse matrix $S M_{J^{1}}^{-1}$, where $J^{1}$ is the set of indices of all columns of the submatrix. For convenience of presentation and without loss of generality, we can assume that $J^{1}=\left\{1, \ldots,\left|I^{1}\right|+1\right\}$. We consider the point $x^{1}=x^{0}+x^{2}$, where $x^{0}$ is an inner point of $F, x^{2}=\left(x_{1}^{2}, \ldots, x_{n}^{2}\right)^{T}$ determined by

$$
\begin{aligned}
& x_{j}^{2}= \begin{cases}d_{j} \beta^{0} & \text { if } 1 \leq j \leq\left|I^{1}\right|+1, \\
0 & \text { if }\left|I^{1}\right|+1<j \leq n,\end{cases} \\
& N\left(J^{1}\right)=\left\{i \in \bar{I}_{\max } \mid \sum_{j \in J^{1}} a_{i j} d_{j}<0\right\}, \\
& \beta^{0}=\max \left\{\left(b_{i}-a_{i} x^{0}\right) / \sum_{j \in J^{1}} a_{i j} d_{j} \mid i \in N\left(J^{1}\right)\right\}
\end{aligned}
$$

if $N\left(J^{1}\right) \neq \varnothing$ and $\beta^{0}$ is an arbitrary negative number if $N\left(J^{1}\right)=\varnothing$. Since $x^{0}$ is an inner point of $F, \beta^{0}<0$. It can be easily seen that $x^{1}$ satisfies the conditions $A\left(I^{1}\right) x^{1}=b\left(I^{1}\right), \quad a_{i} x^{1}=\beta^{0}+b_{i^{0}}$ and $A\left(\bar{I}_{\max }\right) x^{1} \leqslant b\left(\bar{I}_{\max }\right)$. Hence, it easily follows that $x^{1} \in S\left(I^{1}, I^{2}\right)$ and $x^{1} \notin S\left(I_{\max }, \bar{I}_{\max }\right)$. This is a contradiction because $S\left(I^{1}, I^{2}\right)=S\left(I_{\max }, \bar{I}_{\max }\right)$. Therefore, $r\left(I^{1}\right)=r\left(I_{\max }\right)$ and $I^{1} \in T_{1}\left(F, I_{\max }\right)$.

Consequently, since $\left(I^{1}, I^{2}\right) \in R E_{\text {prim }}\left(F, I_{\max }\right)$, there is $G^{1} \subseteq \bar{I}_{\max }$ such that $I^{2}=$ $\bar{I}_{\max } \backslash G^{1}$. It is clear that $S\left(I_{\max }, \bar{I}_{\max }\right)=S\left(I_{\max }, \bar{I}_{\max } \backslash G^{1}\right)$ and $\bar{I}_{\max } \backslash G^{1}$ does not contain any redundant indices for $S\left(I_{\max }, \bar{I}_{\max } \backslash G^{1}\right)$. We will show that $a_{j}^{*}\left(I_{\max }, \bar{I}_{\max } \backslash G^{1}\right) \leqslant b_{j}$ for all $j \in G^{1}$. Assume that there are $j^{0} \in G^{1}$ and $x^{0} \in S\left(I_{\max }, \bar{I}_{\max } \backslash G^{1}\right)$ such that $a_{j^{0}} x^{0}>$ $b_{j^{0}}$. It is clear that $x^{0} \notin S\left(I_{\max }, \bar{I}_{\max } \backslash\left\{G^{1} \backslash\left\{j^{0}\right\}\right\}\right)$. Hence, we have $S\left(I_{\max }, \bar{I}_{\max }\right) \subseteq$ $S\left(I_{\max }, \bar{I}_{\max } \backslash\left\{G^{1} \backslash\left\{j^{0}\right\}\right\}\right) \subset S\left(I_{\max }, \bar{I}_{\max } \backslash G^{1}\right)$. Noting that $S\left(I_{\max }, \bar{I}_{\max }\right)=S\left(I_{\max }, \bar{I}_{\max } \backslash\right.$ $\left.G^{1}\right)$, we have $S\left(I_{\max }, \bar{I}_{\max } \backslash\left\{G^{1} \backslash\left\{j^{0}\right\}\right\}\right)=S\left(I_{\max }, \bar{I}_{\max } \backslash G^{1}\right)$. This is a contradiction. Therefore, we have $a_{j}^{*}\left(I_{\max }, \bar{I}_{\max } \backslash G^{1}\right) \leqslant b_{j}$ for all $j \in G^{1}$. Thus, $G^{1} \in T_{2}\left(F, I_{\max }\right)$. 
From the definition of $T_{3}\left(F, I_{\max }\right)$ and since $\bar{I}_{\max } \backslash G^{1}$ does not contain any redundant indices for $S\left(I_{\max }, \bar{I}_{\max } \backslash G^{1}\right)$, it follows that $G^{1} \in T_{3}\left(F, I_{\max }\right)$. Therefore, $R E^{1}\left(F, I_{\max }\right) \supseteq$ $R E_{\text {prim }}\left(F, I_{\max }\right)$. The proof is complete.

From the definition of minimal representations of a face, Theorems 2.4 and 3.4, the following corollary can be easily obtained:

Corollary 3.5 $|J|=|M|$ and $|K|=|N|$ for all $(J, K) \in R E_{\min }(F)$ and $(M, N) \in$ $R E_{\min }(F)$.

From the proof of Theorem 3.4, the following property is easily obtained:

Property 3.6 If $G \subseteq K$, then $S(J, K)=S(J, K \backslash G)$ if and only if $a_{j}^{*}(J, K \backslash G) \leq b_{j}$ for all $j \in G$.

Remark 3.7 From Theorems 2.4 and 3.4, it can be seen that a face can have many minimal representations.

It is easily seen that $j \in L$ is a strongly redundant index for $S(K, L)$ if and only if $a_{j}^{*}(K, L \backslash\{j\})<b_{j}$. Based on Theorems 2.4 and 3.4, we have a method for determining all minimal representations of a face. In order to increase the usefulness of the method, we will prove and utilize a property that deleting strongly redundant indices for $S(J, \bar{J})$ does not change the redundancy of other indices, where $J$ is an element of $R E(F)$.

Remark 3.8 From the definition of redundant indices and Property 3.2, it follows that $S(J, \bar{J} \backslash\{j\})=F$ for all $j \in \bar{J}^{1}$. Therefore, $\max \left\{a_{j} x \mid x \in F\right\}<b_{j}$ for all $j \in \bar{J}^{1,1}$ and $\max \left\{a_{j} x \mid x \in F\right\}=b_{j}$ for all $j \in \bar{J}^{1,2}$.

We consider the following property:

Property 3.9 (i) If $\bar{J}^{1,1} \neq \emptyset$, then $S(J, \bar{J} \backslash\{i, j\})=F$ for all $i \in \bar{J}^{1,1}$ and $j \in \bar{J}^{1}$. (ii) If $\bar{J}^{1,2} \neq \emptyset$, then $S\left(J, \bar{J} \backslash\left\{\bar{J}^{1,1} \cup\{j\}\right\}\right)=F$ for every $j \in \bar{J}^{1,2}$.

Proof (i) It can easily be seen that

$$
\begin{aligned}
S(J, \bar{J} \backslash\{i, j\}) & =\left\{x \in S(J, \bar{J} \backslash\{i, j\}) \mid a_{i} x \leq b_{i}\right\} \bigcup\left\{x \in S(J, \bar{J} \backslash\{i, j\}) \mid a_{i} x>b_{i}\right\} \\
& =S(J, \bar{J} \backslash\{j\}) \bigcup\left\{x \in S(J, \bar{J} \backslash\{i, j\}) \mid a_{i} x>b_{i}\right\} \\
& =F \bigcup\left\{x \in S(J, \bar{J} \backslash\{i, j\}) \mid a_{i} x>b_{i}\right\} .
\end{aligned}
$$

Noting that

$$
F=\left\{\begin{array}{l|c}
x \in \mathbb{R}^{n} \mid \begin{array}{c}
a_{t} x=b_{t}, t \in J, \\
a_{t} x \leq b_{t}, t \in \bar{J} \backslash\{i, j\}, \\
a_{i} x \leq b_{i}
\end{array}
\end{array}\right\},
$$

$\max \left\{a_{i} x \mid x \in F\right\}<b_{i}$ (Remark 3.8) and $a_{i} x$ is a continuous function on $S(J, \bar{J} \backslash\{i, j\})$, we easily have $S(J, \bar{J} \backslash\{i, j\}) \subseteq\left\{x \in \mathbb{R}^{n} \mid a_{i} x \leq b_{i}\right\}$. Therefore, $\left\{x \in S(J, \bar{J} \backslash\{i, j\}) \mid a_{i} x>b_{i}\right\}=\emptyset$ and $S(J, \bar{J} \backslash\{i, \bar{j}\})=F$. 
(ii) If $\bar{J}^{1,1}=\emptyset$, then the proof is obvious. If $\bar{J}^{1,1} \neq \emptyset$, we consider the set $G=\bar{J}^{1,1} \cup\{j\}$. It can be written that $S(J, \bar{J} \backslash G)=\left\{x \in S(J, \bar{J} \backslash G) \mid a_{i} x \leq b_{i}\right\} \cup$ $\left\{x \in S(J, \bar{J} \backslash G) \mid a_{i} x>b_{i}\right\}=S\left(J, \bar{J} \backslash\left\{\bar{J}^{1,1} \backslash\{i\}\right\} \cup\{j\}\right) \bigcup\left\{x \in S(J, \bar{J} \backslash G) \mid a_{i} x>b_{i}\right\}$, where $i \in \bar{J}^{1,1}$. By induction on the number of elements of $\bar{J}^{1,1}$, we have $\left\{x \in S(J, \bar{J} \backslash G) \mid a_{i} x \leq b_{i}\right\}=F$ and by an argument similar to that presented in part (i) we have $\left\{x \in S(J, \bar{J} \backslash G) \mid a_{i} x>b_{i}\right\}=\emptyset$, the proof of part (ii) can be easily obtained.

By an analogous proof to the proof in part (ii) of Property 3.9, the following result is easily obtained:

Corollary 3.10 If $\bar{J}^{1,2}=\emptyset$, then $S\left(J, \bar{J} \backslash \bar{J}^{1,1}\right)=F$.

From Property 3.9 and Theorem 3.4, we can easily obtain the following corollary:

Corollary 3.11 If $\left|\bar{I}_{\max }^{1}\right| \leq 2$ and $\bar{I}_{\max }^{1,1} \neq \emptyset$, then $R E_{\text {prim }}\left(F, I_{\max }\right)=\left\{\left(J, \bar{I}_{\max }^{2}\right) \mid J \in\right.$ $\left.T_{1}\left(F, I_{\max }\right)\right\}$.

\section{An Algorithm for Determining All Minimal Representations of a Given Face}

Let $S O\left(j, I_{\max }, G\right)$ be the set of all new feasible solutions of the problem $P\left(j, I_{\max }, \bar{I}_{\max } \backslash\right.$ $G)$ that have been found in determining

$$
\begin{aligned}
& a_{j}^{*}\left(I_{\max }, \bar{I}_{\max } \backslash G\right) ; \\
& S O\left(I_{\max }\right)=\bigcup\left\{S O\left(j, I_{\max }, G\right) \mid a_{j}^{*}\left(I_{\max }, \bar{I}_{\max } \backslash G\right) \text { has been determined }\right\} ; \\
& S O\left(I_{\max }, G\right)=\left\{y \in S O\left(I_{\max }\right) \mid a_{j} y \leq b_{j}, \forall j \in \bar{I}_{\max } \backslash G\right\} ; \\
& \Omega^{1}=\left\{G \subseteq \bar{I}_{\max }^{1} \mid \exists j \in G: a_{j}^{*}\left(I_{\max }, \bar{I}_{\max } \backslash G\right)>b_{j} \text { and has been determined }\right\} ;
\end{aligned}
$$

$T G^{t}$ be the set of subsets of $\bar{I}_{\text {max }}^{1}$ that need to be checked for finding elements of $T_{2}\left(F, I_{\max }\right)$ in the $t$-th iteration; $T_{4}\left(F, I_{\max }\right)$ be the set of all maximal elements by inclusion among elements of $T_{2}\left(F, I_{\max }\right)$ that have been found.

An algorithm for finding all minimal representations of the face $F$ described by an index set $I$ is stated as follows:

Step 1 Determine the sets $I_{\max }, T_{1}\left(F, I_{\max }\right)$.

If $\bar{I}_{\max }=\emptyset$, then set $T_{4}\left(F, I_{\max }\right)=\emptyset$ and go to Step 9 .

Set $t=1, T G^{1}=\left\{S \subseteq \bar{I}_{\max }|| S \mid=1\right\}, T_{4}\left(F, I_{\max }\right)=\emptyset, \Omega^{1}=\emptyset, \bar{I}_{\max }^{1,1}=\emptyset$, $\bar{I}_{\max }^{1,2}=\emptyset, S O\left(I_{\max }\right)=\emptyset$ and go to Step 3 .

Step 2 Determine $\bar{I}_{\max }^{1}$.

If $\bar{I}_{\max }^{1}=\emptyset$, then set $T_{4}\left(F, I_{\max }\right)=\emptyset$ and go to Step 9 .

Set $T_{4}\left(F, I_{\max }\right)=\left\{\bar{I}_{\text {max }}^{1,1} \cup\{j\} \mid j \in \bar{I}_{\text {max }}^{1,2}\right\}$.

If $\left|\bar{I}_{\max }^{1}\right|=1$, then go to Step 9 . 
If $\left|\bar{I}_{\max }^{1}\right| \leq 2$ and $\bar{I}_{\max }^{1,1} \neq \varnothing$, then go to Step 9 .

Set $t=2, T G^{t+1}=\emptyset$ and $T G^{t}=\left\{S \subseteq \bar{I}_{\max }^{1}|| S \mid=t\right\}$.

Step 3 Take $G \in T G^{t}$.

If there is $\Omega \in T_{4}\left(F, I_{\max }\right)$ such that $G \subseteq \Omega$, then go to Step 7 .

Set $H=G$.

Step 4 If $S O\left(I_{\max }, G\right)=\emptyset$, then take an arbitrary element $j^{0} \in H$, determine $a_{j^{0}}^{*}\left(I_{\max }, \bar{I}_{\max } \backslash G\right)$ and go to Step 5 .

Find an index $j^{0} \in H$ and a feasible solution $x^{*}$ determined by

$$
a_{j^{0}} x^{*}=\max \left\{\max \left\{a_{j} x \mid x \in S O\left(I_{\max }, G\right)\right\} \mid j \in H\right\} .
$$

Determine $a_{j^{0}}^{*}\left(I_{\max }, \bar{I}_{\max } \backslash G\right)$ based on solving the problem $P\left(j^{0}, I_{\max }, \bar{I}_{\max } \backslash G\right)$ starting from $x^{*}$.

Step 5 If $a_{j^{0}}^{*}\left(I_{\max }, \bar{I}_{\max } \backslash G\right)>b_{j^{0}}$, then set

$$
\Omega^{1}=\Omega^{1} \cup\{G\} \text { and go to Step } 8 .
$$

If $t=1$ and $a_{j^{0}}^{*}\left(I_{\max }, \bar{I}_{\max } \backslash G\right)<b_{j^{0}}$, then set $\bar{I}_{\max }^{1,1}=\bar{I}_{\max }^{1,1} \cup\left\{j^{0}\right\}$.

If $t=1$ and $a_{j^{0}}^{*}\left(I_{\max }, \bar{I}_{\max } \backslash G\right)=b_{j^{0}}$, then set $\bar{I}_{\text {max }}^{1,2}=\bar{I}_{\max }^{1,2} \cup\left\{j^{0}\right\}$.

If $S O\left(j^{0}, I_{\max }, G\right) \neq \emptyset$, then set

$$
S O\left(I_{\max }, G\right)=S O\left(I_{\max }, G\right) \cup S O\left(j^{0}, I_{\max }, G\right) .
$$

Step 6 Set $H=H \backslash\left\{j^{0}\right\}$.

If $H \neq \emptyset$, then go to Step 4 .

If $S\left(I_{\max }, G\right) \neq \emptyset$, then set $S O\left(I_{\max }\right)=S O\left(I_{\max }\right) \cup S O\left(I_{\max }, G\right)$.

If $t=1$, then go to Step 8 .

Let $T_{4}^{1}\left(F, I_{\max }, G\right)=\left\{\Omega \in T_{4}\left(F, I_{\max }\right) \mid \Omega \subseteq G\right\}$ and set

$$
T_{4}\left(F, I_{\max }\right)=T_{4}\left(F, I_{\max }\right) \backslash T_{4}^{1}\left(F, I_{\max }, G\right) \cup\{G\} .
$$

If $t=\left|\bar{I}_{\text {max }}^{1}\right|$, then go to Step 8 .

Step 7 Let $T G_{1}^{t+1}(G)=\left\{G \cup\{i\} \mid i \in \bar{I}_{\text {max }}^{1} \backslash G\right\}$,

$$
T G_{2}^{t+1}(G)=\left\{\Omega \in T G^{t+1} \cup T G_{1}^{t+1}(G) \mid \exists S \in \Omega^{1}: S \subseteq \Omega\right\}
$$

and set

$$
T G^{t+1}=T G^{t+1} \cup T G_{1}^{t+1}(G) \backslash T G_{2}^{t+1}(G) .
$$

Step 8 Set $T G^{t}=T G^{t} \backslash\{G\}$.

If $T G^{t} \neq \emptyset$, then go to Step 3 .

If $t=1$, then go to Step 2 .

If $T G^{t+1} \neq \emptyset$, then set $t=t+1, T G^{t+1}=\emptyset$ and go to Step 3 . 
Step $9 R E_{\min }(F)=\left\{\left(K, \bar{I}_{\max } \backslash G\right) \mid K \in T_{1}\left(F, I_{\max }\right), G \in T_{4}\left(F, I_{\max }\right)\right\}$.

Step 10 Stop.

Now, we present some properties of the algorithm.

Property 4.1 (i) If $\Omega \in T_{2}\left(F, I_{\max }\right)$ and $G \subseteq \Omega$, then $G \in T_{2}\left(F, I_{\max }\right)$.

(ii) If $\Omega \notin T_{2}\left(F, I_{\max }\right)$ and $\Omega \subseteq G$, then $G \notin T_{2}\left(F, I_{\max }\right)$.

(iii) If $\bar{I}_{\max }^{1} \neq \emptyset$, then $S O\left(I_{\max }, G\right) \neq \emptyset$ for all $G \in T G^{t}$ and $t \geq 2$.

Proof (i) Since $G \subseteq \Omega, S\left(I_{\max }, \bar{I}_{\max } \backslash G\right) \subseteq S\left(I_{\max }, \bar{I}_{\max } \backslash \Omega\right)$. From this and $\Omega \in$ $T_{2}\left(F, I_{\max }\right)$, it follows that $a_{j}^{*}\left(I_{\max }, \bar{I}_{\max } \backslash G\right) \leq a_{j}^{*}\left(I_{\max }, \bar{I}_{\max } \backslash \Omega\right) \leq b_{j}$ for all $j \in G$. Therefore, $G \in T_{2}\left(F, I_{\max }\right)$.

(ii) The proof is immediately obtained from part (i).

(iii) From the algorithm, it follows that there is $j^{0} \in \bar{I}_{\max }^{1}$ such that $\left\{j^{0}\right\} \subseteq G$. Thus, $\bar{I}_{\max } \backslash G \subseteq \bar{I}_{\max } \backslash\left\{j^{0}\right\}$. From the definition of $S O\left(I_{\max }, G\right)$, it follows that $x^{o p t}\left(j^{0},\left\{j^{0}\right\}\right) \in$ $S O\left(I_{\max }, G\right)$, where $x^{o p t}\left(j^{0},\left\{j^{0}\right\}\right)$ is an optimal solution of the problem $P\left(j^{0}, I_{\max }, \bar{I}_{\max } \backslash\right.$ $\left.\left\{j^{0}\right\}\right)$ and has been found in checking $\left\{j^{0}\right\}$ to be an element of $\bar{I}_{\max }^{1}$. The proof is complete.

Corollary 4.2 If $\Omega \notin T_{2}\left(F, I_{\max }\right)$, then at least $2^{\left|\bar{I}_{\max }^{1} \backslash \Omega\right|}$ subsets of $\bar{I}_{\max }^{1}$ are not elements of $T_{2}\left(F, I_{\max }\right)$.

Proof From part (ii) of Property 4.1, it follows that $\Omega \cup \Omega^{*} \notin T_{2}\left(F, I_{\max }\right)$ for all $\Omega^{*} \subseteq$ $\bar{I}_{\max }^{1} \backslash \Omega$. It is easily seen that the number of such sets $\Omega^{*}$ is equal to $2^{\left|\bar{I}_{\max }^{1} \backslash \Omega\right|}$. The proof is complete.

Remark 4.3 Part (iii) of Property 4.1 is a basis of choosing a feasible solution $x^{*}$ in Step 4. The algorithm contains many ideas to reduce the computational efforts for determining the set $R E_{\min }(F)$. Some of them are shown below.

- Parts (i) and (ii) of Properties 4.1 are utilized by introducing and using rules (4.1), (4.3), and (4.6) to reduce the number of problems of type (3.2) that need to be solved for checking subsets of $\bar{I}_{\max }^{1}$ to be elements of $T_{2}\left(F, I_{\max }\right)$.

- For determining $a_{j^{0}}^{*}\left(I_{\max }, \bar{I}_{\max } \backslash G\right)$ in Step 4, solving the problem $P\left(j^{0}, I_{\max }, \bar{I}_{\max } \backslash G\right)$ can be started from a best feasible solution among its feasible solutions that have been found by using rule (4.2). In addition, the problem $P\left(j^{0}, I_{\max }, \bar{I}_{\max } \backslash G\right)$ is not required to solve to optimality if there is $x^{0} \in S\left(I_{\max }, \bar{I}_{\max } \backslash G\right)$ such that $a_{j^{0}} x^{0}>b_{j^{0}}$.

- It can be easily seen that the feasible sets of the problems $P\left(j, I_{\max }, \bar{I}_{\max } \backslash G\right)$ are the same for all $j \in G$ and $S\left(I_{\max }, \bar{I}_{\max } \backslash \Omega\right) \subseteq S\left(I_{\max }, \bar{I}_{\max } \backslash G\right)$ if $\Omega \subseteq G$. These properties are utilized to reduce computational efforts in determining $a_{j^{0}}^{*}\left(I_{\max }, \bar{I}_{\max } \backslash G\right)$ by introducing and using by the rules (4.1)-(4.4).

The validity of the algorithm is dealt with in the following property:

Property 4.4 The set $R E_{\min }(F)$ is obtained after the final iteration of the algorithm. 
Proof From Theorems 2.4 and 3.4, it is enough to prove that $T_{4}\left(F, I_{\max }\right)=T_{3}\left(F, I_{\max }\right)$. If $\bar{I}_{\max }^{1}=\emptyset$, then the proof is obvious. Now, we present the proof in the case when $\bar{I}_{\max }^{1} \neq \varnothing$. Let $t^{1}$ be a maximal integer number such that $T G^{t^{1}} \neq \emptyset$. We consider an arbitrary subset $G$ of $\bar{I}_{\text {max }}^{1}$ and will show that if $G \notin \bigcup\left\{T G^{t} \mid t=1, \ldots, t^{1}\right\}$, then $G \notin T_{2}\left(F, I_{\max }\right)$. Since $G \notin \bigcup\left\{T G^{t} \mid t=1, \ldots, t^{1}\right\}$, there is $S \in \Omega^{1}$ such that $S \subseteq G$. Noting that $S \notin T_{2}\left(F, I_{\max }\right)$, from Property 4.1 , it follows that $G \notin T_{2}\left(F, I_{\max }\right)$. Thus, we have $T_{2}\left(F, I_{\max }\right) \subseteq \bigcup\left\{T G^{t} \mid t=1, \ldots, t^{1}\right\}$. It can be easily seen that all elements of $T_{2}\left(F, I_{\max }\right)$ are found by the algorithm and $T_{4}\left(F, I_{\max }\right) \subseteq T_{2}\left(F, I_{\max }\right)$. In addition, from rule (4.5) and from a property of top-down search procedures that $|G|<|H|$ for all $G \in T G^{t}$ and $H \in T G^{l}$ with $t<l$, it follows that the set $T_{4}\left(F, I_{\max }\right)$ consists of all maximal elements of $T_{2}\left(F, I_{\max }\right)$ ordered by the inclusion. Therefore, from the definition of $T_{3}\left(F, I_{\max }\right)$, we have $T_{4}\left(F, I_{\max }\right)=T_{3}\left(F, I_{\max }\right)$.

Remark 4.5 The algorithm can also efficiently find all minimal representations of the face $F$ described by an index set $I$ of a large-scale convex polyhedron (the number of equalities and inequalities used to state a polyhedron is large) with some computational notes that the set $I_{\max }$ is found based on the problems of type (3.1); Gaussian elimination is used to compute the rank of matrices in determining the set $T_{1}\left(F, I_{\max }\right)$ and decomposition methods, interior point methods or Lagrangian methods [5] can be used to solve large-scale LP problems constructed in the algorithm.

\section{Examples}

Example 5.1 Determine all minimal representations of the face $F$ described by index set $I=\emptyset$ of polyhedron (1.1), when

$$
A=\left(\begin{array}{llllllll}
-1 & -2 & -3 & 0 & -1 & 1 & 1 \\
-1 & -1 & -1 & 0 & 1 & 1 & -1 \\
1 & 2 & 3 & 1 & 1 & 1 & 1
\end{array}\right)^{T}
$$

and $b=\left(\begin{array}{lllllll}1 & 2 & 3 & 2 & 1 & 1 & 1\end{array}\right)^{T}$.

To illustrate the working of the algorithm, in this example, the simplex method is used to solve problems of type (3.2).

Step $1 \quad I_{\max }=\emptyset, T_{1}\left(F, I_{\max }\right)=\emptyset$.

$t=1, T G^{1}=\{\{1\},\{2\},\{3\},\{4\},\{5\},\{6\},\{7\}\}, T_{4}\left(F, I_{\max }\right)=\emptyset, \Omega^{1}=\emptyset, \bar{I}_{\max }^{1,1}=\emptyset$, $\bar{I}_{\max }^{1,2}=\emptyset, S O\left(I_{\max }\right)=\emptyset$.

Step 3 Take $G=\{1\} . H=\{1\}$.

Step $4 S O\left(I_{\max },\{1\}\right)=\emptyset$, take $j^{0}=1$.

$S O\left(1, I_{\max },\{1\}\right)=\left\{(0,0,0)^{T},(0,0,1)^{T},(-.3333,-1.3333,0)^{T}\right\}, a_{1}^{*}\left(I_{\max }, \bar{I}_{\max } \backslash\{1\}\right)=$ 2.

Step $5 a_{1}^{*}\left(I_{\max }, \bar{I}_{\max } \backslash\{1\}\right)>b_{1}, \Omega^{1}=\{\{1\}\}$.

Step $6 H=\emptyset . S O\left(I_{\max }\right)=\left\{(0,0,0)^{T},(0,0,1)^{T},(-.3333,-1.3333,0)^{T}\right\}$.

Step $8 T G^{1}=\{\{2\},\{3\},\{4\},\{5\},\{6\},\{7\}\}$.

Step 3 Take $G=\{2\} . H=\{2\}$.

Step $4 S O\left(I_{\max },\{2\}\right)=\left\{(0,0,0)^{T},(0,0,1)^{T}\right\}, j^{0}=2, x^{*}=(0,0,1)^{T} . x^{*}$ is an optimal solution of problem $P\left(2, I_{\max }, \bar{I}_{\max } \backslash\{2\}\right), a_{2}^{*}\left(I_{\max }, \bar{I}_{\max } \backslash\{2\}\right)=2$. 
Step $5 a_{2}^{*}\left(I_{\max }, \bar{I}_{\max } \backslash\{2\}\right)=b_{2}, \bar{I}_{\max }^{1,2}=\{\{2\}\} . S O\left(2, I_{\max },\{2\}\right)=\emptyset$.

Step $6 \quad H=\emptyset$.

Step $8 T G^{1}=\{\{3\},\{4\},\{5\},\{6\},\{7\}\}$.

Step 3 Take $G=\{3\}$. $H=\{3\}$.

Step $4 S O\left(I_{\max },\{3\}\right)=\left\{(0,0,0)^{T},(0,0,1)^{T}\right\}, j^{0}=3, x^{*}=(0,0,1)^{T} . x^{*}$ is an optimal solution of problem $P\left(3, I_{\max }, \bar{I}_{\max } \backslash\{3\}\right), a_{3}^{*}\left(I_{\max }, \bar{I}_{\max } \backslash\{3\}\right)=3$.

Step $5 a_{3}^{*}\left(I_{\max }, \bar{I}_{\max } \backslash\{3\}\right)=b_{3}, \bar{I}_{\max }^{1,2}=\{\{2\},\{3\}\} . S O\left(3, I_{\max },\{3\}\right)=\emptyset$.

Step $6 \quad H=\emptyset$.

Step $8 T G^{1}=\{\{4\},\{5\},\{6\},\{7\}\}$.

Step 3 Take $G=\{4\}$. $H=\{4\}$.

Step $4 S O\left(I_{\max },\{4\}\right)=\left\{(0,0,0)^{T},(0,0,1)^{T}\right\}, j^{0}=4, x^{*}=(0,0,1)^{T} . x^{*}$ is an optimal solution of problem $P\left(4, I_{\max }, \bar{I}_{\max } \backslash\{4\}\right), a_{4}^{*}\left(I_{\max }, \bar{I}_{\max } \backslash\{4\}\right)=1$.

Step $5 a_{4}^{*}\left(I_{\max }, \bar{I}_{\max } \backslash\{4\}\right)<b_{4}, \bar{I}_{\max }^{1,1}=\{\{4\}\} . S O\left(4, I_{\max },\{4\}\right)=\emptyset$.

Step $6 H=\emptyset$.

Step $8 T G^{1}=\{\{5\},\{6\},\{7\}\}$.

Step 3 Take $G=\{5\} . H=\{5\}$.

Step $4 S O\left(I_{\max },\{5\}\right)=\left\{(0,0,0)^{T},(0,0,1)^{T}\right\}, j^{0}=5, x^{*}=(0,0,1)^{T}$.

$S O\left(5, I_{\max },\{5\}\right)=\left\{(-2,3,0)^{T}\right\}, a_{5}^{*}\left(I_{\max }, \bar{I}_{\max } \backslash\{5\}\right)=2$.

Step $5 a_{5}^{*}\left(I_{\max }, \bar{I}_{\max } \backslash\{5\}\right)>b_{5}, \Omega^{1}=\{\{1\},\{5\}\}$.

Step $6 H=\emptyset . S O\left(I_{\max }\right)=\left\{(0,0,0)^{T},(0,0,1)^{T},(-.3333,-1.3333,0)^{T},(-2,3,0)^{T}\right\}$.

Step $8 T G^{1}=\{\{6\},\{7\}\}$.

Step 3 Take $G=\{6\} . H=\{6\}$.

Step $4 S O\left(I_{\max },\{6\}\right)=\left\{(0,0,0)^{T},(0,0,1)^{T}\right\}, j^{0}=6, x^{*}=(0,0,1)^{T}$.

$P\left(6, I_{\max }, \bar{I}_{\max } \backslash\{6\}\right)$ is unbounded from above, $a_{6}^{*}\left(I_{\max }, \bar{I}_{\max } \backslash\{6\}\right)=2$.

Step $5 a_{6}^{*}\left(I_{\max }, \bar{I}_{\max } \backslash\{6\}\right)>b_{6}, \Omega^{1}=\{\{1\},\{5\},\{6\}\}$.

Step $6 H=\emptyset$.

Step $8 T G^{1}=\{\{7\}\}$.

Step 3 Take $G=\{7\} . H=\{7\}$.

Step $4 S O\left(I_{\max },\{7\}\right)=\left\{(0,0,0)^{T},(0,0,1)^{T}\right\}, j^{0}=7, x^{*}=(0,0,1)^{T}$.

$P\left(7, I_{\max }, \bar{I}_{\max } \backslash\{7\}\right)$ is unbounded from above, $a_{7}^{*}\left(I_{\max }, \bar{I}_{\max } \backslash\{7\}\right)=2$.

Step $5 a_{7}^{*}\left(I_{\max }, \bar{I}_{\max } \backslash\{7\}\right)>b_{7}, \Omega^{1}=\{\{1\},\{5\},\{6\},\{7\}\}$.

Step $6 H=\emptyset$.

Step $8 T G^{1}=\emptyset$.

Step $2 \bar{I}_{\max }^{1}=\{\{2\},\{3\},\{4\}\}$,

$S O\left(I_{\max }\right)=\left\{(0,0,0)^{T},(0,0,1)^{T},(-.3333,-1.3333,0)^{T},(-2,3,0)^{T}\right\} . T_{4}\left(F, I_{\max }\right)=$ $\{\{2,4\},\{3,4\}\} . t=2, T G^{3}=\emptyset, T G^{2}=\{\{2,3\},\{2,4\},\{3,4\}\}$.

Step 3 Take $G=\{2,3\} . H=\{2,3\}$.

Step $4 S O\left(I_{\max },\{2,3\}\right)=\left\{(0,0,0)^{T},(0,0,1)^{T}\right\}, j^{0}=3, x^{*}=(0,0,1)^{T} . x^{*}$ is an optimal solution of problem $P\left(3, I_{\max }, \bar{I}_{\max } \backslash\{2,3\}\right), a_{3}^{*}\left(I_{\max }, \bar{I}_{\max } \backslash\{2,3\}\right)=b_{3}$, $S O\left(\{3\}, I_{\max },\{2,3\}\right)=\emptyset$.

Step $6 H=\{2\}$.

Step $4 S O\left(I_{\max },\{2,3\}\right)=\left\{(0,0,0)^{T},(0,0,1)^{T}\right\}, j^{0}=2, x^{*}=(0,0,1)^{T} . x^{*}$ is an optimal solution of problem $P\left(2, I_{\max }, \bar{I}_{\max } \backslash\{2,3\}\right), a_{2}^{*}\left(I_{\max }, \bar{I}_{\max } \backslash\{2,3\}\right)=b_{2}$, $S O\left(\{2\}, I_{\max },\{2,3\}\right)=\emptyset$.

Step $6 H=\emptyset, T_{4}^{1}\left(F, I_{\max },\{2,3\}\right)=\emptyset, T_{4}\left(F, I_{\max }\right)=\{\{2,3\},\{2,4\},\{3,4\}\}$.

Step $7 T G_{1}^{3}(\{2,3\})=\{\{2,3,4\}\}, T G_{2}^{3}(\{2,3\})=\emptyset, T G^{3}=\{\{2,3,4\}\}$. 
Step $8 T G^{2}=\{\{2,4\},\{3,4\}\}$.

Step 3 Take $G=\{2,4\}$. $G \in T_{4}\left(F, I_{\max }\right)$.

Step $7 T G_{1}^{3}(\{2,4\})=\{\{2,3,4\}\}, T G_{2}^{3}(\{2,4\})=\emptyset, T G^{3}=\{\{2,3,4\}\}$.

Step $8 T G^{2}=\{\{3,4\}\}$.

Step 3 Take $G=\{3,4\} . G \in T_{4}\left(F, I_{\max }\right)$.

Step $7 T G_{1}^{3}(\{3,4\})=\{\{2,3,4\}\}, T G_{2}^{3}(\{3,4\})=\emptyset, T G^{3}=\{\{2,3,4\}\}$.

Step $8 T G^{2}=\emptyset . T G^{3}=\{\{2,3,4\}\}, t=3, T G^{4}=\emptyset$.

Go on the same way, we obtain that $T_{4}\left(F, I_{\max }\right)=\{\{2,3,4\}\}, T G^{4}=\emptyset$.

Step $9 R E_{\min }(F)=\{(\emptyset,\{1,5,6,7\})\}$.

Step 10 Stop.

Example 5.2 Find all minimal representations of the face $F$ described by index set $I=\emptyset$ of polyhedron (1.1) when

$$
A=\left(\begin{array}{llllll}
1 & -1 & 0 & 0 & 1 & 1 \\
0 & 0 & 1 & -1 & -1 & 1
\end{array}\right)^{T}
$$

and $b=\left(\begin{array}{llllll}0 & 0 & 0 & 0 & 0 & 0\end{array}\right)^{T}$.

This example is also considered in Boneh et al. [2].

Step $1 I_{\max }=\{1,2,3,4,5,6\}, r\left(I_{\max }\right)=2, T_{1}\left(F, I_{\max }\right)=\{\{1,3\},\{1,4\},\{1,5\}\} \bigcup$ $\{\{1,6\},\{2,3\},\{2,4\},\{2,5\},\{2,6\},\{3,5\},\{3,6\},\{4,5\},\{4,6\},\{5,6\}\}, \bar{I}_{\max }=\emptyset$, $T_{4}\left(F, I_{\max }\right)=\emptyset$.

Step $9 R E_{\min }(F)=\{(\{1,3\}, \emptyset),(\{1,4\}, \emptyset),(\{1,5\}, \emptyset),(\{1,6\}, \emptyset),(\{2,3\}, \emptyset),\} \bigcup$ $\{(\{2,4\}, \emptyset),(\{2,5\}, \emptyset),(\{2,6\}, \emptyset),(\{3,5\}, \emptyset),(\{3,6\}, \emptyset),(\{4,5\}, \emptyset),(\{4,6\}, \emptyset),(\{5,6\}, \emptyset)\}$. Step 10 Stop.

Remark 5.3 In Example 5.2, the face $F$ has 13 minimal representations in which only one minimal representation $(\{1,3\}, \emptyset)$ is shown in Boneh et al. [2].

\section{Some Applications of Minimal Representations of a Face}

A subset $H$ of a face $G$ is said to be a sub-face of it if $H$ is a face of the polyhedron $G$. The number of constraints used to represent a face is said to be $a$ size of the face. Let $N C(F,(M, N))$ be the size of the face $F$ represented by an index pair $(M, N)$. We consider the following property:

Property 6.1 (i) $N C(F,(I, \bar{I}))-N C(F,(J, K))=m-r\left(I_{\max }\right)-|K|$ for all $(J, K) \in$ $R E_{\min }(F)$.

(ii) $N C(F,(J, K))=N C(F,(M, N))$ for all $(J, K) \in R E_{\min }(F)$ and $(M, N) \in$ $R E_{\min }(F)$.

Proof (i) From Theorems 2.4 and 3.4, it follows that $(J, K)$ is a weak reduction of $\left(I_{\max }, \bar{I}_{\max }\right)$. Therefore, there are $G^{1} \subseteq I_{\max }$ and $G^{2} \subseteq \bar{I}_{\max }$ such that $(J, K)=$ $\left(I_{\max } \backslash G^{1}, \bar{I}_{\max } \backslash G^{2}\right)$. Thus, $N C(F,(I, \bar{I}))-N C(F,(J, K))=(|I|+|\bar{I}|)-\left(\left|I_{\max } \backslash G^{1}\right|+\right.$ $\left.\left|\bar{I}_{\max } \backslash G^{2}\right|\right)=(|I|+|\bar{I}|)-\left(\left|I_{\max }\right|-\left|G^{1}\right|+\left|\bar{I}_{\max }\right|-\left|G^{2}\right|\right)=\left|G^{1}\right|+\left|G^{2}\right|$. From Theorems 2.4 and 3.4, it follows that $|J|=r\left(I_{\max }\right)$. Therefore, $\left|G^{1}\right|+\left|G^{2}\right|=\left(\left|I_{\max }\right|-|J|\right)+\left(\left|\bar{I}_{\max }\right|-|K|\right)$ $=m-r\left(I_{\max }\right)-|K|$.

(ii) The proof is immediately obtained from Corollary 3.5. 
Property 6.1 shows that the size of the face $F$ is decreased by $m-r\left(I_{\max }\right)-|K|$, if $F$ is represented by a minimal representation $(J, K)$ of it.

Now, we deal with the degeneracy of a face. It is clear that the face $F$ is degenerate if $I_{\max } \neq I$ but it can also happen that $F$ is degenerate when $I_{\max }=I$. A necessary and sufficient condition for the degeneracy of a face is shown in the following property whose proof can be easily obtained on the basis of [19, Property 7.1]:

Property 6.2 The face $F$ represented by a descriptor index set $I$ is degenerate if and only if $r\left(I_{\max }\right)<\left|I_{\max }\right|$.

A degeneracy degree of a face $F$ represented by an index pair $(J, K)$ is denoted by $d(F,(J, K))$ and is computed by the formula

$$
d(F,(J, K))=\left|J_{\max }(K)\right|-r\left(J_{\max }(K)\right),
$$

where $J_{\max }(K)=\left\{i \in J \cup K \mid a_{i} x=b_{i}\right.$ for all $\left.x \in S(J, K)\right\}$. It is easily seen that $J_{\max }(K)=J \cup i q(J, K)$ and the index set $i q(J, K)$ can be easily found by solving $|K|$ linear programming problems $\min \left\{a_{j} x \mid x \in S(J, K)\right\}$, where $j \in K$ or by solving one LP problem similarly constructed as in [19]. The concept of the degeneracy degree of a face is a generalization of that of the degeneracy degree of constraint collections introduced by Sierksma and Tijssen [15]. The face represented by an index pair $(J, K)$ is degenerate if and only if $d(F,(J, K))>0$. A dimension of the face $G$ represented by an index pair $(M, N)$ is denoted by $\operatorname{dim} G$ and is computed by $\operatorname{dim} G=n-r\left(M_{\max }(N)\right.$ ) (see [18] for more details). A face can be represented by an arbitrary descriptor index set for it or by an arbitrary minimal representation of it but the degeneracy degree of the face depends on which are chosen to represent it. This is dealt with in the following property:

Property 6.3 (i) $d(F,(M, \bar{M}))=d(F,(N, \bar{N}))$ for all $M \in R E(F)$ and $N \in R E(F)$.

(ii) If $(M, N) \in T(F),(K, L) \in T(F)$ and $(K, L)$ is a weak reduction of $(M, N)$, then $d(F,(K, L)) \leq d(F,(M, N))$.

(iii) $d(F,(J, K))=0$ for all $(J, K) \in R E_{\min }(F)$.

(iv) $d(F,(J, K))=\min \{d(F,(M, N)) \mid(M, N) \in T(F)\}$ for every $(J, K) \in R E_{\min }(F)$.

Proof (i) From [19, Theorem 3.2], we have $M_{\max }(\bar{M})=I_{\max }$ for all $M \in R E(F)$. Thus $d(F,(M, \bar{M}))=\left|I_{\max }\right|-r\left(I_{\max }\right)$. Therefore, $d(F,(M, \bar{M}))=d(F,(N, \bar{N}))$ for all $M \in$ $R E(F)$ and $N \in R E(F)$.

(ii) It is clear that $K \subseteq M$ and $L \subseteq N$. Noting that $S(K, L)=F=$ $S(M, N)$ and $L \subseteq N$, from the definition of implicit equality indices it follows that $i q(K, L) \subseteq i q(M, N)$. Thus, $K_{\max }(L)=K \cup i q(K, L) \subseteq M \cup i q(M, N)=$ $M_{\max }(N)$. Therefore, $K_{\max }(L) \subseteq M_{\max }(N)$. Since $S(K, L)=F=S(M, N)$, from the definition of the dimension of a face, it follows that $n-r\left(K_{\max }(L)\right)=$ $n-r\left(M_{\max }(N)\right)$. Therefore, $r\left(K_{\max }(L)\right)=r\left(M_{\max }(N)\right)$. Hence, $d(F,(K, L))=$ $\left|K_{\max }(L)\right|-r\left(K_{\max }(L)\right) \leq\left|M_{\max }(N)\right|-r\left(M_{\max }(N)\right)=d(F,(M, N))$. Thus, we have $d(F,(K, L)) \leq d(F,(M, N))$.

(iii) From Theorems 2.4 and 3.4, it follows that $r(J)=|J|$. Since $K$ contains no implicit equality indices for $S(J, K), J_{\max }(K)=J$. Therefore, $d(F,(J, K))=\left|J_{\max }(K)\right|-$ $r\left(J_{\max }(K)\right)=|J|-|J|=0$.

(iv) The proof is immediately obtained from part (iii) by noting that $d(F,(M, N)) \geq 0$ for all $(M, N) \in T(F)$. 
From the proof of Property 6.3, the following property is easily obtained:

Property 6.4 $r\left(K_{\max }(L)\right)=r\left(M_{\max }(N)\right)$ for all $(K, L) \in T(F)$ and $(M, N) \in T(F)$.

Remark 6.5 It can happen that there is $(K, M) \in R E_{\text {prim }}(F)$ such that $d(F,(K, M))>0$. This can be shown by the following example:

Example 6.6 We consider polyhedron (1.1) when

$$
A=\left(\begin{array}{lll}
-1 & 1 & 0 \\
0 & 1 & 1
\end{array}\right)^{T}
$$

$b=\left(\begin{array}{lll}0 & 1 & 1\end{array}\right)^{T}$ and compute the degeneracy degree of the face $F$ consisting of only one point $(0,1)^{T}$. It is easily seen that $(\{3\},\{1,2\})$ is a prime representation of the face $F,\{3\}_{\max }(\{1,2\})=\{1,2,3\}$ and $r\left(\{3\}_{\max }(\{1,2\})\right)=2$. Therefore, $d(F,(\{3\},\{1,2\}))$ $=\left|\{3\}_{\max }(\{1,2\})\right|-r\left(\{3\}_{\max }(\{1,2\})\right)=3-2=1$.

Thus, from Example 6.6 and Property 6.3, it follows that the set $R E_{\min }(F)$ can be a strict subset of the set $R E_{\text {prim }}(F)$.

The degeneracy degree of a face generally depends on descriptor index pairs used to represent it. To show a dependence of the degeneracy degree of a face on descriptor index pairs for it, we say that the face $F$ is degenerate with respect to a descriptor index pair $(M, N)$ if it is represented by $(M, N)$ and $d(F,(M, N))>0$, and is not degenerate with respect to a descriptor index pair $(M, N)$ if it is represented by $(M, N)$ and $d(F,(M, N))=$ 0 . The following property is immediately obtained from Property 6.3:

Property 6.7 The face $F$ is not degenerate with respect to $(J, K)$ for all $(J, K) \in$ $R E_{\min }(F)$.

From Example 6.6, it is easily seen that this property might not be true for prime representations of $F$. Noting that every face of $P$ has at least one minimal representation, from Property 6.7, it follows that for an arbitrary face of $P$, there is always a descriptor index pair for it such that this face is not degenerate with respect to the descriptor index pair. The degeneracy of some special sub-faces of $F$ can be immediately obtained from [15, Theorem 4]:

Property 6.8 If a face is represented by a minimal representation of it and has dimension $d$, then all the $(d-1)$ or $(d-2)$-dimensional sub-faces of it are not degenerate.

In order to show some applications of minimal representations of a face, we consider a problem whose feasible set is described by a face and will investigate the problem when this face is represented by a minimal representation of it. From Properties 6.1 and 6.3, it is easily seen that the degeneracy degree of the face and the size of the problem (the number of the objectives and constraints used to state the problem) can be reduced. These can be used to reduce computational efforts and difficulties in solving and analyzing the problem. Using a minimal representation of a face to represent it gives us special advantages in many methods, for example, face search methods, face decomposition-based methods, 
descriptor set-based methods (methods are based on descriptor sets for faces), etc. In solving a problem of optimizing a function over the efficient set of a multiple objective linear programming (MOLP) problem by face search methods or by descriptor set-based methods (see, e.g., $[1,13,17])$ and in finding the efficient set or determining all maximal efficient faces of an MOLP problem by top-down search methods (see, e.g., [12, 19, 21]), the number of descriptor sets for faces of the constraint polyhedron that need to be considered can be reduced if the constraint polyhedron of the MOLP problem is represented by a minimal representation of it. In addition, based on [19, Property 2.9] and Properties 6.7-6.8, the method in [19] can be improved on the basis of representing the constraint polyhedron by a minimal representation of it because the maximal descriptor index sets for all descriptor sets whose dimensions are elements of the set $\{n,(n-1),(n-2)\}$ need not be determined for this method, where $n$ is the dimension of the constraint polyhedron (the constraint polyhedron is a special face of it) and the dimension of a descriptor set is one of the face described by it (see [19] or [20] for more details). The bottom-up search method given in [20] is also a descriptor set-based method for finding all maximal efficient faces of an MOLP problem. A main computational effort of this method lies in determining the set of all edges and extreme rays incident to every efficient extreme point of the MOLP problem. From Property 6.8 , it can be easily seen that the degeneracy degree of every efficient extreme point can be decreased; and therefore, computational efforts in the method given in [20] can also be decreased by using a minimal representation of the constraint polyhedron to represent this polyhedron.

\section{Conclusions}

A method for determining all minimal representations of a face of a polyhedron is proposed. The maximal descriptor index set for a face dealt with in our previous papers is used to construct the method. To reduce computational efforts in finding all minimal representations of a face, we prove and use properties that deleting strongly redundant constraints does not change the redundancy of other constraints and all minimal representations of the face can be determined by finding only the set of all prime representations of the face corresponding to the maximal descriptor index set for it. The proposed method is based on a top-down search strategy for finding all minimal representations of a face. This method is simple, is easy to implement, and has many computational advantages. Based on minimal representations of a face, a reduction of the degeneracy degree of the face and ideas to improve some known methods for solving a problem for optimizing a function over the efficient set and for finding all maximal efficient faces in multiple objective linear programming are presented.

Acknowledgements I would like to thank the anonymous referee and Editors for their helpful and valuable comments which greatly improved the presentation of the paper.

Funding Open access funding provided by Corvinus University of Budapest.

Open Access This article is licensed under a Creative Commons Attribution 4.0 International License, which permits use, sharing, adaptation, distribution and reproduction in any medium or format, as long as you give appropriate credit to the original author(s) and the source, provide a link to the Creative Commons licence, and indicate if changes were made. The images or other third party material in this article are included in the article's Creative Commons licence, unless indicated otherwise in a credit line to the material. If material is not included in the article's Creative Commons licence and your intended use is not permitted by statutory 
regulation or exceeds the permitted use, you will need to obtain permission directly from the copyright holder. To view a copy of this licence, visit http://creativecommons.org/licenses/by/4.0/.

\section{References}

1. Benson, H.P., Sayin, S.: A face search heuristic algorithm for optimizing over the efficient set. Naval Res. Log. 40, 103-116 (1993)

2. Boneh, A., Caron, R.J., Lemire, F.W., McDonald, J.F., Telgen, J., Vorst, A.C.F.: Note on prime representations of convex polyhedral sets. J. Optim. Theory Appl. 61, 137-142 (1989)

3. Ciripoi, D., Löhne, A., Weißing, B.: Calculus of convex polyhedra and polyhedral convex functions by utilizing a multiple objective linear programming solver. Optimization 68, 2039-2054 (2019)

4. Dam, A.A.T.: Similarity Transformations between Minimal Representations of Convex Polyhedral Sets. NLR Technical Publication NLR TP 93393 (1993)

5. Evtushenko, Y.G., Golikov, A.I., Mollaverdy, N.: Augmented Lagrangian method for large-scale linear programming problems. Optim. Methods Softw. 20, 515-524 (2005)

6. Fukuda, K., Gartner, B., Szedlak, M.: Combinatorial redundancy detection. Ann. Oper. Res. 34, 315 328 (2015)

7. Greenberg, H.J.: Consistency, redundancy, and implied equalities in linear systems. Ann. Math. Artif. Intell. 17, 37-83 (1996)

8. Klintberg, E., Nilsson, M., Mardh, L.J., Gupta, A.: A primal active-set minimal-representation algorithm for polytopes with application to invariant-set calculations. In: IEEE Conference on Decision and Control (2018). https://doi.org/10.1109/CDC.2018.8619642

9. Luan, N.N., Yen, N.D.: A representation of generalized convex polyhedra and applications. Optimization 69(3), 471-492 (2020)

10. Marechal, A., Perin, M.: Efficient elimination of redundancies in polyhedra by raytracing. In: International Conference on Verification, Model Checking and Abstract Interpretation (2017)

11. Rockafellar, R.: Convex Analysis. Princeton University Press, Princeton (1972)

12. Sayin, S.: An algorithm based on facial decomposition for finding the efficient set in multiple objective linear programming. Oper. Res. Lett. 19, 87-94 (1996)

13. Sayin, S.: Optimization over the efficient set using a top-down search of faces. Oper. Res. 48, 65-72 (2000)

14. Scholl, C., Disch, S., Pigorsch, F., Kupferschmid, S.: Computing optimized representations for nonconvex polyhedra by detection and removal of redundant linear constraints. In: TACAS, LNCS, vol. 5505, pp. 383-397. Springer (2009)

15. Sierksma, G., Tijssen, G.A.: Degeneracy degrees of constraint collections. Math. Methods Oper. Res. 53, 437-448 (2003)

16. Telgen, J.: Minimal representation of convex polyhedral sets. J. Optim. Theory Appl. 38, 1-24 (1982)

17. Tu, T.V.: Optimization over the efficient set of a parametric multiple objective linear programming problem. Eur. J. Oper. Res. 122, 570-583 (2000)

18. Tu, T.V.: A common formula to compute the efficient sets of a class of multiple objective linear programming problems. Optimization 64, 2065-2092 (2015)

19. Tu, T.V.: The maximal descriptor index set for a face of a convex polyhedral set and some applications. J. Math. Anal. Appl. 429, 395-414 (2015)

20. Tu, T.V.: A new method for determining all maximal efficient faces in multiple objective linear programming. Acta Math. Vietnam. 42, 1-25 (2017)

21. Yu, P.L., Zeleny, M.: The set of all nondominated solutions in linear cases and a multicriteria simplex method. J. Math. Anal. Appl. 49, 430-468 (1975)

Publisher's Note Springer Nature remains neutral with regard to jurisdictional claims in published maps and institutional affiliations. 